\title{
Robust Control and Fuzzy Logic Guidance for an Unmanned Surface Vehicle
}

\author{
Marcelo M. Huayna-Aguilar ${ }^{1}$, Juan C. Cutipa-Luque ${ }^{2}$, Pablo Raul Yanyachi ${ }^{3}$ \\ Electronic Engineering Professional School ${ }^{1}$ \\ Universidad Nacional de San Agustín de Arequipa, Av. Venezuela s/n, Cercado, Arequipa, Peru \\ Pedro Paulet's Astronomic and Aerospace Institute ${ }^{2,3}$ \\ Universidad Nacional de San Agustín de Arequipa, Cerro San Francisco s/n, Characato, Arequipa, Peru
}

\begin{abstract}
This work deals with guidance and control of an unmanned surface vehicle which has the mission to monitor autonomously the water quality condition in Peruvian sea onshore. The vehicle is a catamaran class with two slender bodies propelled by two electric thrusts in differential and common modes in order to maneuver in surge and in yaw directions. A multivariable control approach is proposed in order to control these two variables and a fuzzy logic-based guidance tracks predefined trajectories at the sea surface. The conjunction between robust control and guidance algorithms is validated numerically and the results show good stability and performance despite the presence of disturbance, noise sensors and model uncertainties.
\end{abstract}

Keywords-Robust control; guidance; fuzzy; unmanned surface vehicle

\section{INTRODUCTION}

The growth, industrialization and concentration of mass population in metropolitan cities have produced risks to vulnerable areas. The Peruvian big cities are close to the Pacific sea, lagoons and rivers, vulnerable to the human activity or natural disasters. Therefore, water quality monitoring is vital for understanding the dynamics of these water bodies. Peruvian public institutions monitor regularly the ocean and lagoons using manned craft and ships through local instrumentation, remote sensing and sampled data analysis [1]. These activities demand steady and high financial support and the use of unmanned surface vehicles (USVs) is becoming more attractive for research institutions due to low-cost, autonomy, and to reduce human risks.

Nowadays, there are many unmanned surface vehicles operating in the world. In [2], the authors present the DELFIM USV designed to carry out mission tasks of data acquisition of marine environment and to serve as a communication hub between a terrain base and multiple autonomous underwater vehicles. The DELFIM has integrated guidance, navigation and control to carry out straightforward its tasks. In [3], the CaRoLime USV presents integration between electronic hardware in order to execute missions for limnology studies in ocean and rivers. The paper also presents a mathematical model obtained using system identification approach and least square estimation optimization. In 2017, the Shanghai University has developed an USV to carry out validation of algorithms applied to hydrography of coastal areas [4]. There are more information about the development and the application of these vehicles that can be found in [5].

The unmanned vehicles is commonly designed with an architecture composed of embedded electronic hardware and software to make possible its maneuvering autonomously or semi-autonomously. The guidance and control algorithms allow the vehicle to move in maritime environment following predefined trajectories set remotely or through an embedded digital memory. The control system of these vehicles should guarantee better performance and stability despite the presence of disturbances, electrical sensor noise and model uncertainties. There are many robust control strategies applied to these unmanned maritime vehicles. An $\mathcal{H}_{\infty}$ robust mixed sensitivity approach is applied to an autonomous underwater vehicle in [6], an $\mathcal{H}_{2}$ robust control approach is applied to an autonomous underwater vehicle (AUV) characterized by output disturbances and time delay in [7], a nonlinear robust control is applied to other unmanned maritime vehicle for hull ship inspection in [8], and another modified robust control algorithm for an USV is proposed in [9].

Relative to the guidance, the work of [10] has been a source of inspiration for nowadays trends. A robust guidance control, based in $\mathcal{H}_{\infty}$ control and Fuzzy guidance, is proposed in [11] to carry out three-dimensional inspection maneuvers of an AUV. Other authors propose a waypoint guidance using fuzzy logic to generate controller command signals [12]. In [13], the authors proposed an improvement to the classical lineof-sight (LOS) guidance algorithm with and integral action and adapting to different cruise speeds. Path following based in LOS is still an active research area to evaluate the USV performance subject to disturbance, noise sensor, parameter variation, and nonlinear constraints imposed by actuators and other unmodeled dynamics [14], [15], [16].

This paper deals with $\mathcal{H}_{\infty}$ robust control and fuzzy logic guidance system for the EDSON-J vehicle, an USV of the UNSA (Universidad Nacional de San Agustín de Arequipa). A multivariable and centralized controller is synthesized using mixed sensitivity approach and a guidance system based on Mamdani type fuzzy logic rule. The main contribution of this paper is to combine the robustness properties of an advanced controller synthesized according to frequency domain requirement with a conventional guidance algorithm based on LOS. The organization of this paper follows: section I presents the introduction over guidance and control in unmanned maritime vehicles, section II presents the mathematical model representation of the EDSON-J USV, section III presents the robust and multivariable control approach, section IV presents the guidance based on fuzzy and LOS approaches, section V presents the numerical results using a simulator and section 
VI provides the conclusions.

\section{EDSON-J MODEL}

The EDSON-J is a catamaran class vehicle, which is under development at the UNSA since 2019 to carry out inspection and monitoring tasks in the Pacific sea coastal and lagoons of Arequipa (south region of Peru country). The vehicle has a length of $3 \mathrm{~m}$ and width of $1.6 \mathrm{~m}$, draft and free-board of $0.6 \mathrm{~m}$.

The unmanned vehicle has been modeled using the rigid body dynamics and hydrodynamics forces interaction between the hull structure and the fluid according to the nomenclature given in [17]. Fig. 1 shows the EDSON-J USV reference frames, an inertial-frame fixed at earth and other body-frame attached at the geometric center of the vehicle. The inertialframe considers three longitudinal position $(x, y, z)$ and three angular displacements $(\phi, \theta, \psi)$, the body-frame considers three linear velocities $(u, v, w)$ and three angular velocities $(p, q, r)$. Unlike underwater vehicles, it is assumed the USV has motions constrained to the water surface and are neglected heave $z$, roll $\phi$ and pitch $\theta$ motions. Then, the EDSON-J nonlinear model can be summarized in kinematic and dynamic equations, respectively:

$$
\begin{gathered}
\dot{\eta}=J \nu, \\
M \dot{\nu}+C(\nu) \nu+D(\nu) \nu=b \tau,
\end{gathered}
$$

where the $\eta=\left[\begin{array}{lll}x & y & \psi\end{array}\right]^{T}$ is the earth frame position vector, the $\nu=\left[\begin{array}{lll}u & v & r\end{array}\right]^{T}$ is the body frame velocity vector. The control input vector is representing by $\tau=\left[\begin{array}{ll}n_{c} & n_{d}\end{array}\right]^{T}$ indicating the common mode $n_{c}$ and differential mode $n_{d}$ propeller actions. $J(\eta)$ is the coordinate transformation matrix between the earth and body frames, $M$ is the rigid body mass plus the added mass of the vehicle, $C(\nu)$ includes rigid body and Coriolis force terms, $D(\nu)$ is the damping matrix, $b$ is the coefficient related to the control input $\tau$. These matrices are expressed following:

$$
\begin{gathered}
J(\eta)=\left[\begin{array}{ccc}
\cos \psi & -\sin \psi & 0 \\
\sin \psi & \cos \psi & 0 \\
0 & 0 & 1
\end{array}\right] \\
M=\left[\begin{array}{ccc}
m-X_{\dot{u}} & 0 & 0 \\
0 & m-Y_{\dot{v}} & m x_{G}-Y_{\dot{r}} \\
0 & m x_{G}-Y_{\dot{r}} & I_{z z}-N_{\dot{r}}
\end{array}\right], \\
C(\nu)=\left[\begin{array}{ccc}
0 & C_{12}(\nu) & C_{13}(\nu) \\
C_{21}(\nu) & 0 & C_{33}(\nu) \\
C_{31}(\nu) & C_{32}(\nu) & 0
\end{array}\right] \\
D(\nu)=-\left[\begin{array}{ccc}
D_{11}(\nu) & 0 & 0 \\
0 & D_{22}(\nu) & 0 \\
0 & 0 & D_{33}(\nu)
\end{array}\right]
\end{gathered}
$$

where:

$$
\begin{aligned}
& C_{12}(\nu)=-m r \\
& C_{13}(\nu)=-m x_{G} r+Y_{\dot{v}} v+\frac{Y_{\dot{r}}+N_{\dot{v}}}{2} \\
& C_{21}(\nu)=m r \\
& C_{23}(\nu)=-X_{\dot{u}} u \\
& C_{31}(\nu)=m x_{G} r-Y_{\dot{v}} v-\frac{Y_{\dot{r}}-N_{\dot{v}}}{2} r \\
& C_{32}(\nu)=X_{\dot{u}} u
\end{aligned}
$$

TABLE I. EDSON-J USV NONLINEAR MODEL PARAMETERS.

\begin{tabular}{c|c||c}
\hline Parameter & Value & Unit \\
\hline$m$ & 250 & $\mathrm{~kg}$ \\
$x_{G}$ & 0.08 & $\mathrm{~m}$ \\
$I_{z}$ & 204.1000 & $\mathrm{~kg} / \mathrm{m}^{2}$ \\
$X_{\dot{u}}$ & -2.4706 & $\mathrm{~kg}$ \\
$X_{u}$ & -0.2912 & $\mathrm{~kg} / \mathrm{s}$ \\
$X_{|u| u}$ & -27.6262 & $\mathrm{~kg} / \mathrm{m}$ \\
$Y_{\dot{v}}$ & -247.0649 & $\mathrm{~kg}$ \\
$Y_{v}$ & -123.5324 & $\mathrm{~kg} / \mathrm{s}$ \\
$Y_{\dot{r}}$ & -370.5973 & $\mathrm{~kg} \cdot \mathrm{m}$ \\
$Y_{|v| v}$ & -38.9275 & $\mathrm{~kg} / \mathrm{m}$ \\
$N_{\dot{r}}$ & -748.3102 & $\mathrm{~kg} \cdot \mathrm{m}^{2}$ \\
$N_{\dot{v}}$ & -370.5973 & $\mathrm{~kg} \cdot \mathrm{m}$ \\
$N_{r}$ & -741.1947 & $\mathrm{~kg} \cdot \mathrm{m}^{2} / \mathrm{s}$ \\
$N_{|r| r}$ & -262.7609 & $\mathrm{~kg} \cdot \mathrm{m}^{2}$ \\
\hline \multicolumn{2}{|c}{} \\
$D_{11}(\nu)=X_{u}+X_{|u| u}|u|$, \\
$D_{22}(\nu)=Y_{v}+Y_{|v| v}|v|$, \\
$D_{33}(\nu)=N_{r}+N_{|r| r}|r|$.
\end{tabular}

The EDSON-J model parameters can be obtained using the rigid body dynamics and the slender body theory through empirical and semi-empirical relations [18]. The computed parameter values for the EDSON-J nonlinear model are presented in Table I.

\section{EDSON-J ROBUST CONTROL}

This section presents the multivariable robust control approach based on $\mathcal{H}_{\infty}$ mixed sensitivity. The nonlinear EDSONJ model expressed in (1) and (2) is linearized using Taylor expansion series around the cruise speed of $u=2 \mathrm{~m} / \mathrm{s}$. To guarantee the linear controllability and observability system properties, some states are neglected and the reduced system has the state variables $\mathrm{X}=\left[\begin{array}{ccc}u & v & \dot{\psi}\end{array}\right]^{T}$, control input variable $\mathrm{U}=\left[\begin{array}{ll}n_{c} & n_{d}\end{array}\right]^{T}$ and output variable $\mathrm{Y}=\left[\begin{array}{ll}u & \dot{\psi}\end{array}\right]^{T}$. The linear EDSON-J model is expressed in the state space form as follows:

$$
G:=\left\{\begin{array}{l}
\dot{\mathrm{X}}=\mathrm{AX}+\mathrm{BU} \\
\mathrm{Y}=\mathrm{CX}+\mathrm{DU}
\end{array}\right.
$$

where the matrices are as follows:

$$
\begin{gathered}
A=\left[\begin{array}{ccc}
-0.3294 & 0 & 0 \\
0 & 0.0800 & 0.0858 \\
0 & -2.3951 & -1.5949
\end{array}\right], \\
\mathrm{B}=\left[\begin{array}{cc}
0.2109 & 0 \\
0 & 0.1145 \\
0 & -0.8347
\end{array}\right] \\
\mathrm{C}=\left[\begin{array}{lll}
1 & 0 & 0 \\
0 & 0 & 1
\end{array}\right] \\
\mathrm{D}=\left[\begin{array}{ll}
0 & 0 \\
0 & 0
\end{array}\right]
\end{gathered}
$$




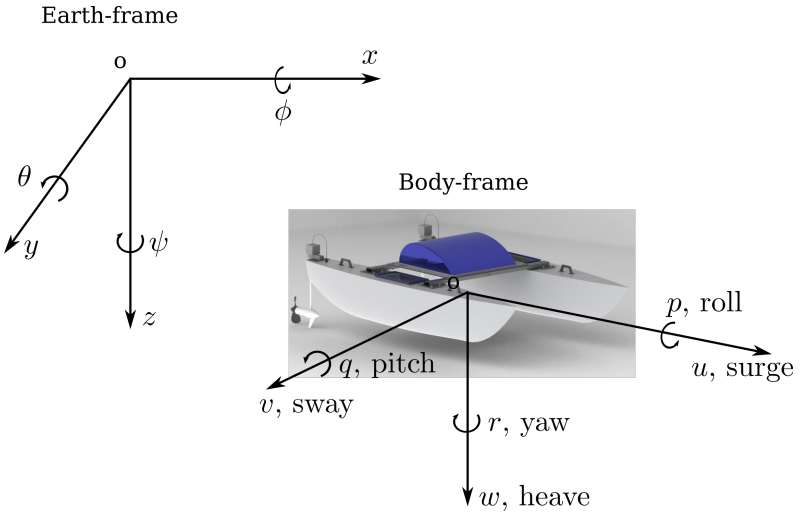

Fig. 1. Coordinate Frames for the EDSON-J vehicle.

Fig. 2 presents the two ports structure used to synthesize the robust controller through mixed sensitivity approach, where $G$ is the linear model, $W_{*}$ are the weighting functions, $R$ is the reference pre-filter and $K$ is the synthesized robust controller. The extended plant $P$ and the controller $K$ form the closed loop system $T_{\mathrm{zw}}$; which has the exogenous input w composed of reference signal $\tilde{r}$, input disturbance $\tilde{d}_{i}$, output disturbance $\tilde{\mathrm{d}}$ and sensor noise $\tilde{n}$; and has the exogenous output $z$ composed of tracking error ẽ and output response $\tilde{y}$.

A closed loop matrix $T_{\mathrm{zw}}$ is measured using $\mathcal{H}_{\infty}$ norm to guarantee good performance and stability [19], [6]:

$$
\left\|T_{\mathrm{zw}}\right\|=\left\|\begin{array}{c}
W_{S} S \\
W_{T} T
\end{array}\right\|_{\infty}
$$

where $S=(I+K G)^{-1}$ is the sensitivity matrix, $T=(I-S)$ is the complementary sensitivity matrix, and $I$ is an identity matrix, $W_{S}$ is the weighting sensitivity matrix and $W_{T}$ is the weighting of complementary sensitivity matrix. Assuming that the matrices involved satisfy necessary detectability and stabilizability conditions, and based on well-known results, there is an optimal controller $K(s)$ so that a closed loop function between $w$ and $z$ satisfies [19]:

$$
\left\|T_{\mathrm{zw}}\right\|_{\infty}=\gamma
$$

where $\gamma$ is a real number associated to a suboptimal control problem.

The $\mathcal{H}_{\infty}$ mixed sensitivity approach was formally used in former work to control an autonomous underwater vehicle [6]. In this paper, the authors uses the same approach applied to the EDSON-J USV where the input pre-filter and weighting matrices $R, W_{i}, W_{d}$ and $W_{n}$ are identity matrices of $I_{2 \times 2}$. The output weighting sensitivity matrices are diagonal matrices:

$$
\begin{gathered}
W_{S}=\operatorname{diag}\left\{\frac{0.5 s+1}{s+0.0001}, \frac{0.5 s+1}{s+0.0001}\right\}, \\
W_{T}=\operatorname{diag}\left\{\frac{s+1}{0.0001 s+5}, \frac{s+1}{0.0001 s+5}\right\} .
\end{gathered}
$$

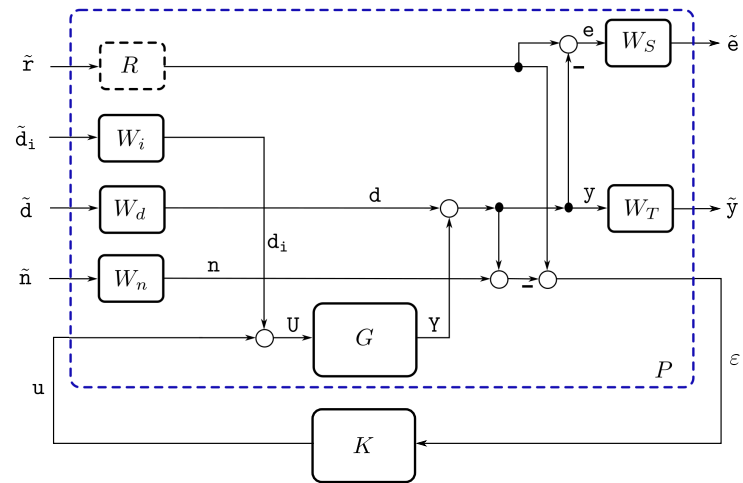

Fig. 2. Two Ports Configuration to $\mathcal{H}_{\infty}$ Synthesis [6].

\section{EDSON-J GUIDANCE}

The structure of the robust guidance control for EDSON-J vehicle is described in Fig. 3 where the robust control guarantees a good tracking of the cruise speed command $u_{d}$ and a good tracking of the yaw rate command $\dot{\psi}_{d}$. This last signal will be generated by the guidance algorithm composed of two subsystems. This section presents the guidance algorithm of the vehicle that integrates the Lookahead-based steering approach and a fuzzy logic controller to generate yaw rate command considering information of LOS.

\section{A. Lookahead-based Steering}

The goal of this subsystem is to generate the steering command (yaw angle) using the definiciton of LOS and Lookahead-based sterring algorithm. The geometric representation of line of sight (LOS) is as shown in Fig. 4, where $(x, y)$ is the actual position of the vehicle, $\left(x_{k}, y_{k}\right)$ and $\left(x_{k+1}, y_{k+1}\right)$ are waypoints that define a straight-line path. The path-tangential angle can be expressed as follows [10]:

$$
\alpha_{k}:=\operatorname{atan} 2\left(y_{k+1}-y_{k}, x_{k+1}-x_{k}\right),
$$

where the atan 2 is the four-quadrant version of arctan constrained to $[-\pi / 2, \pi / 2]$. The USV coordinates in the path-fixed reference frame $\left(x_{e}, y_{e}\right)$ can be written as:

$$
\left[\begin{array}{l}
x_{e} \\
y_{e}
\end{array}\right]=\left[\begin{array}{cc}
\cos (\alpha) & -\sin (\alpha) \\
\sin (\alpha) & \cos (\alpha)
\end{array}\right]^{T}\left[\begin{array}{l}
x-x(k) \\
y-y(k)
\end{array}\right]
$$

where $x_{e}$ is the along-track distance and $y_{e}$ is the cross-track error. If the vehicle follows a path, only the cross-track error is relevant because the vehicle converges to the straight-line when $y_{e} \rightarrow 0$. Considering the second row of expression (19), the cross-track error can be re-arranged as:

$$
y_{e}=-\left(x-x_{k}\right) \sin \left(\alpha_{k}\right)+\left(y-y_{k}\right) \cos \left(\alpha_{k}\right),
$$

After defined the LOS guidance, a lookahead-based algorithm can be expressed as:

$$
\chi_{d}=\alpha_{k}+\arctan \left(\frac{-y_{e}}{\Delta}\right)
$$

where the second term of expression is the velocity-path relative angle, which ensures the velocity towards at point located ahead a distance $\Delta>0$. The angle $\chi_{d}$ is transformed to the yaw angle command $\psi_{d}$ :

$$
\psi_{d}=\chi_{d}-\beta
$$




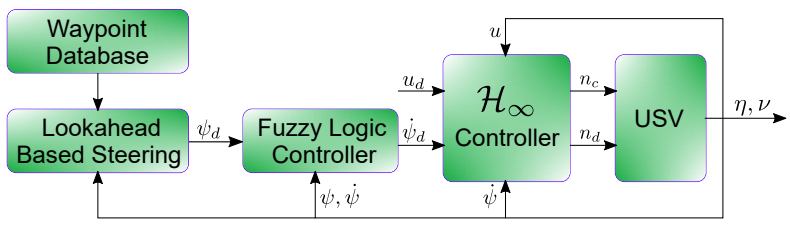

Fig. 3. USV Robust Guidance Control.

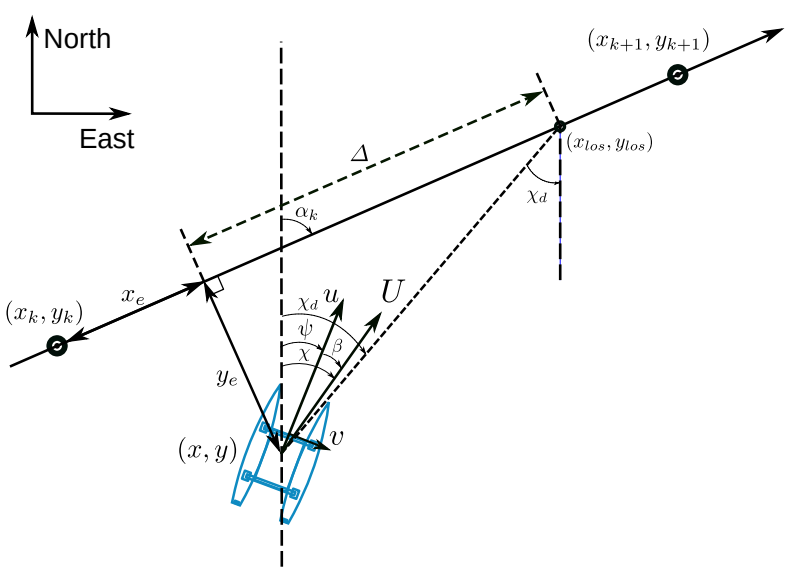

Fig. 4. LOS Guidance Geometry.

where,

$$
\beta=\arcsin \left(\frac{v}{U}\right)
$$

\section{B. Fuzzy Logic Yaw Rate Controller}

The fuzzy logic rule reads the yaw angle command $\psi_{d}$ from the LOS block and the actual yaw angle $\psi$ from the inertial sensor, and computes the error $\tilde{\psi}=\psi_{d}-\psi$ as one input. The second input is the actual yaw rate $\dot{\psi}$ from the inertial sensor. This block uses a Mamdani fuzzy inference to generate the yaw rate command $\dot{\psi}_{d}$ which feeds the robust control system as reference signal (Fig. 3).

Fig. 5 and 6 present the membership functions relative to the two inputs. These inputs use five Gaussian membership function defined as: negative medium NM, negative small NS, zero ZE, positive small PS, and positive medium PM. The one output uses seven triangular membership functions defined as: negative big NB, negative medium NM, negative small NS, zero ZE, positive small PS, positive medium PM, positive big PB (see Fig. 7). It uses the centroid defuzzification which is represented by the surface in Fig. 8 .

\section{RESUlts}

The proposed alternative for good tracking and robust performance is validated numerically using the EDSON-J nonlinear model simulator considering three degree of freedom.

\section{A. Robust Controller}

The suboptimal gamma value achieved is $\gamma=1.0067$ and the value of the $K$ controller is reduced to nine states. Fig. 9 presents the surge sensitivity $S$ response with low value

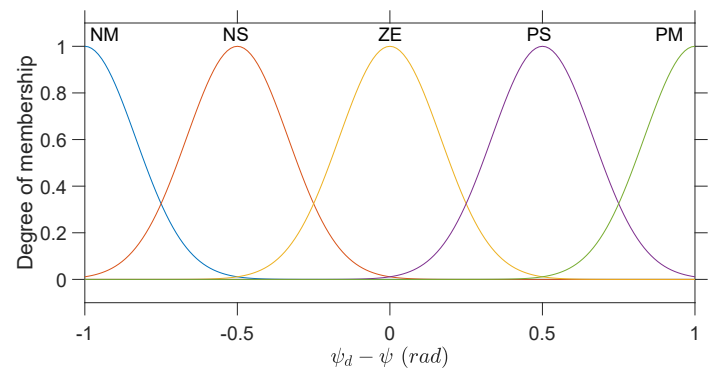

Fig. 5. The Yaw Error $\tilde{\psi}=\psi_{d}-\psi$ Input Membership Functions.

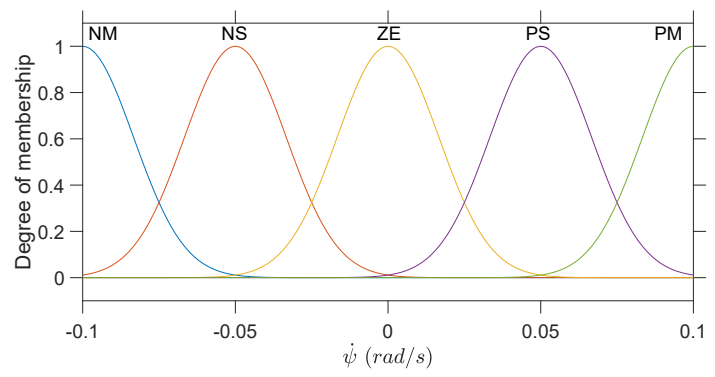

Fig. 6. The Yaw Rate $\dot{\psi}$ Input Membership Functions.

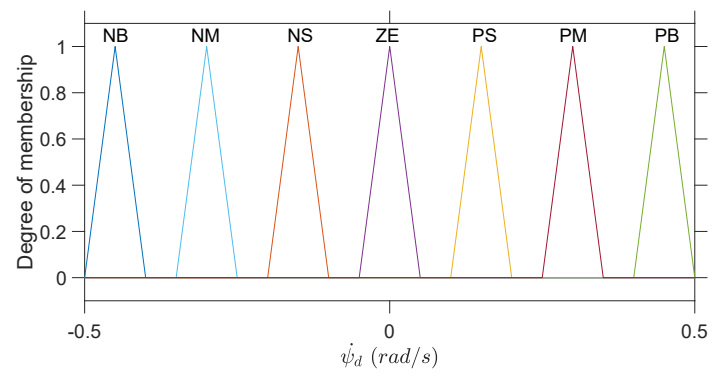

Fig. 7. Output Yaw Rate Desired.

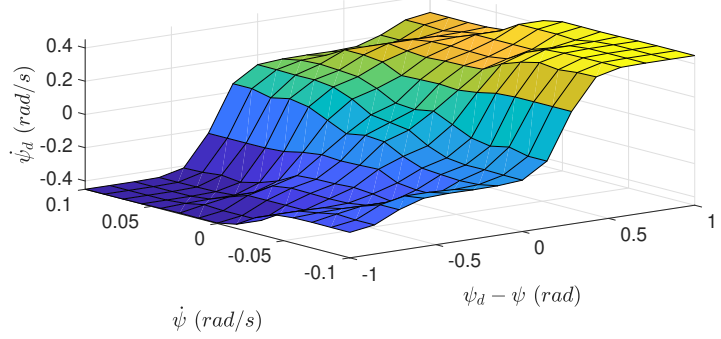

Fig. 8. The Surface of the Fuzzy Inference System. 


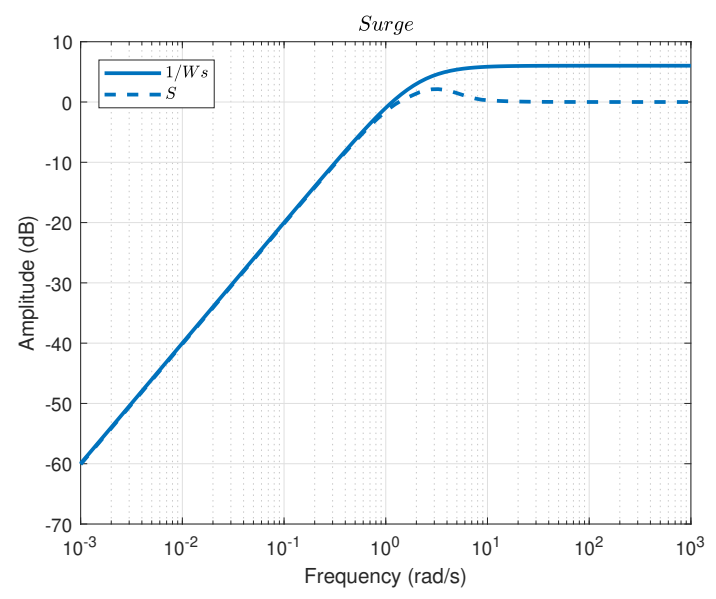

Fig. 9. Sensitivity $S$ and its Weighting Inverse $1 / W_{S}$ for Surge Velocity $u$.

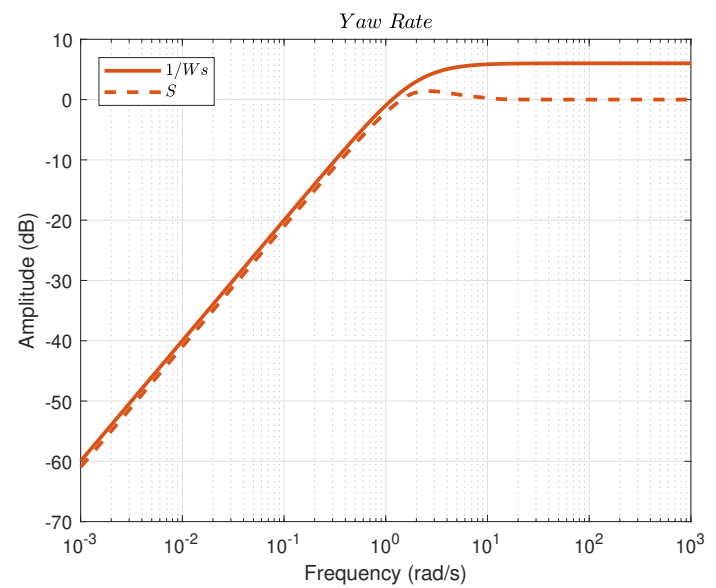

Fig. 10. Sensitivity $S$ and its Weighting Inverse $1 / W_{S}$ for Yaw Rate $\dot{\psi}$.

at low frequencies with slope of $20 \mathrm{~dB} / \mathrm{dec}$ and it does not cross its weighting given by $1 / W_{S}$. Fig. 10 presents the yaw rate sensitivity $S$ response with low value at low frequencies with slope of $20 \mathrm{~dB} / \mathrm{dec}$ and it does not cross its weighting given by $1 / W_{S}$. Both results achieve crossover frequencies of $1.12 \mathrm{rad} / \mathrm{s}$ and is considered as good tracking and rejection to environmental disturbances, such as waves, currents and wind. Fig. 11 presents the surge complementary sensitivity $T$ response with low value at high frequencies with slope of -20 $\mathrm{dB} / \mathrm{dec}$ and it does not cross its weighting given by $1 / W_{T}$. Fig. 12 presents the yaw rate complementary sensitivity $S$ response with low value at high frequencies with slope of $-20 \mathrm{~dB} / \mathrm{dec}$ and it does not cross its weighting given by $1 / W_{T}$. Both results achieve crossover frequencies of $6.2 \mathrm{rad} / \mathrm{s}$ and is considered as good stability and robustness to model uncertainties and noise rejection.

The closed loop matrix given in (14) achieves the desired shaping. Fig. 13 and 14 shown that the $\mathcal{H}_{\infty}$ norm is limited to values less than 1 .

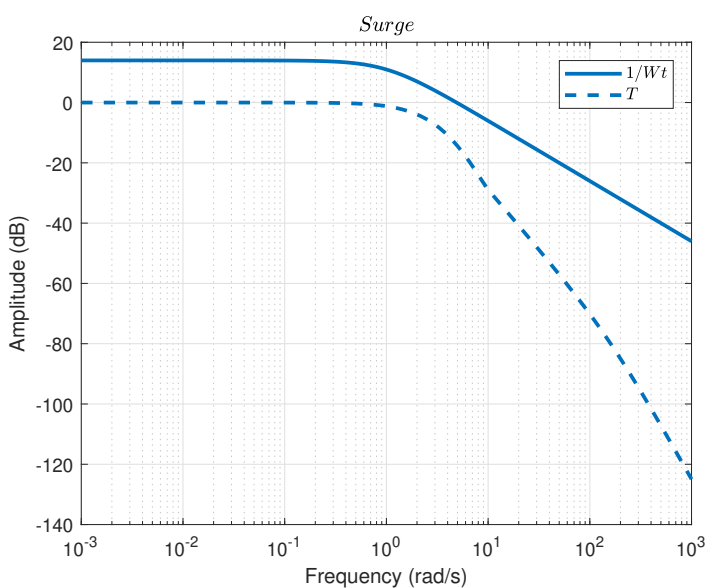

Fig. 11. Complementary Sensitivity $T$ and its Weighting Inverse $1 / W_{T}$ for Surge Velocity $u$.

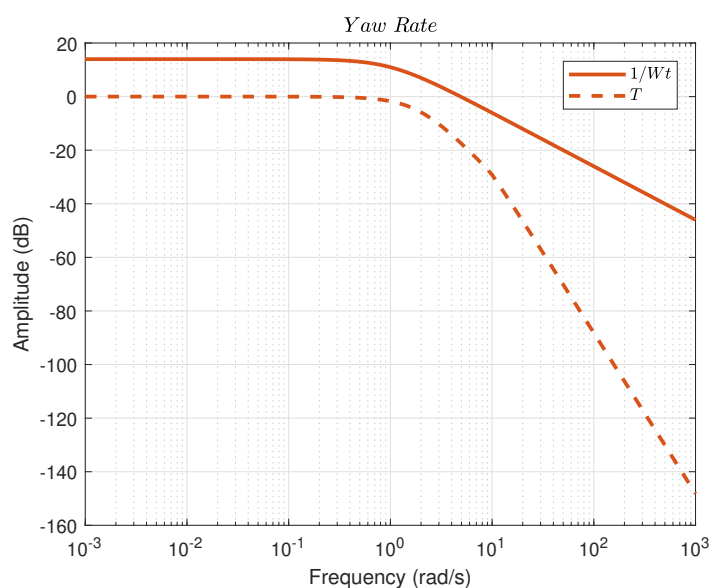

Fig. 12. Complementary Sensitivity $T$ and its Weighting Inverse $1 / W_{T}$ for Yaw Rate $\dot{\psi}$.

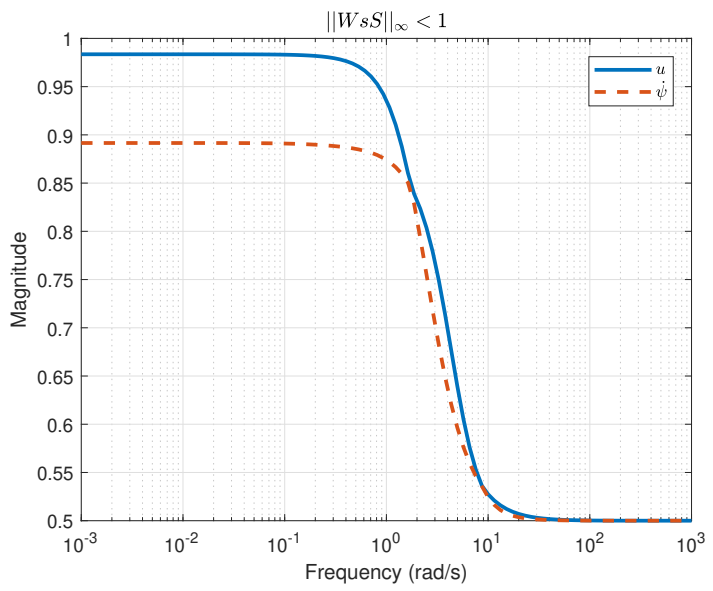

Fig. 13. Transfer Matrix $W_{S} S$. 


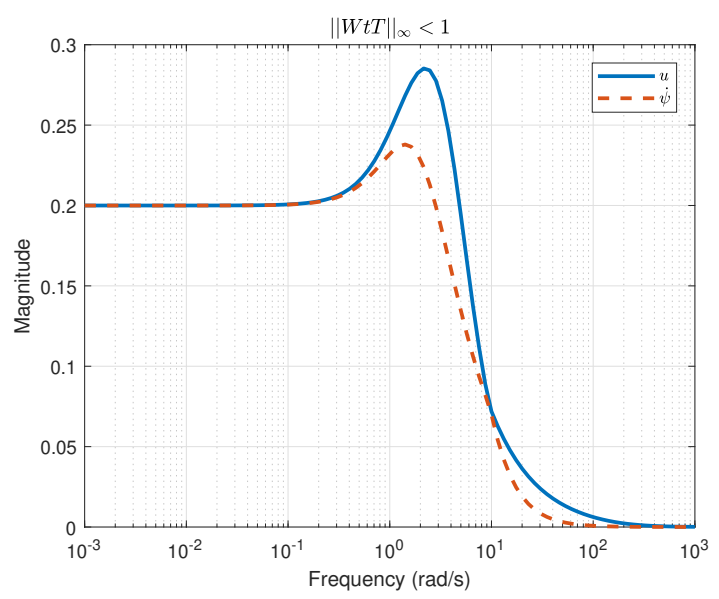

Fig. 14. Transfer Matrix $W_{T} T$.

\section{B. Fuzzy Logic Guidance}

The guidance of EDSON-J is validates with LOS ratio of $5 \mathrm{~m}$ and with zigzag and circular trajectories. The cruise speed command is keeping to $u_{d}=2 \mathrm{~m} / \mathrm{s}$ meanwhile the vehicle follows the desired path and Fuzzy logic controller generates the yaw rate command $\dot{\psi}_{d}$ feeding the Robust controller.

Fig. 15 shows the vehicle follows a desired circular path starting at (0.0) and has a maximum tracking error of 3.52 $\mathrm{m}$, an acceptable value assuming that the LOS ratio is $5 \mathrm{~m}$. The reduction of LOS distance can cause degradation in the Fuzzy logic controller performance and may require more energy consumption of the propellers. Fig. 16 presents the state variables responses $u, v$ and $\dot{\psi}$ during the performance of this path following. The surge velocity $u$ achieves the desired value set by $u_{d}$, the sway velocity $v$ has transitory oscillation due to the fuzzy logic rules and steering command given by Lookahead-base steering algorithm. However, at steady state the oscillatory behavior is limited keeping the vehicle velocity at values of $0.08 \mathrm{~m} / \mathrm{s}$. The way rate response $\dot{\psi}$ has similar oscillatory transition and in steady state goes to a constant value of $0.013 \mathrm{rad} / \mathrm{s}$. These values are suitable considering the circular path with $150 \mathrm{~m}$ of ratio.

Finally, a zigzag path of $20 \mathrm{~m}$ amplitude is considered in Fig. 17. The vehicle follows the desired trajectory with good performance and the maximum tracking error is $0.5 \mathrm{~m}$. Fig. 18 presents the $u, v$ and $\dot{\psi}$ responses during the performance of this path following. The surge velocity $u$ achieves the desired value set by $u_{d}$, the sway velocity $v$ has transitory oscillation due to the fuzzy logic rules and steering command given by Lookahead-base steering algorithm. Unlike the last test, this sway velocity oscillate in order to do the turning maneuver in both directions. The yaw rate response $\dot{\psi}$ has also an oscillatory behavior, a maximum value of $0.48 \mathrm{rad} / \mathrm{s}$ is achieved in transitory response.

The frequency domain analysis of the robust control approach achieves all the specifications for the USV, a multivariable and coupled system. The Fuzzy guidance, in conjunction with the robust controller, guaranteed good tracking of straight and curved trajectories. The time domain responses show good performance without compromising stability and maintaining

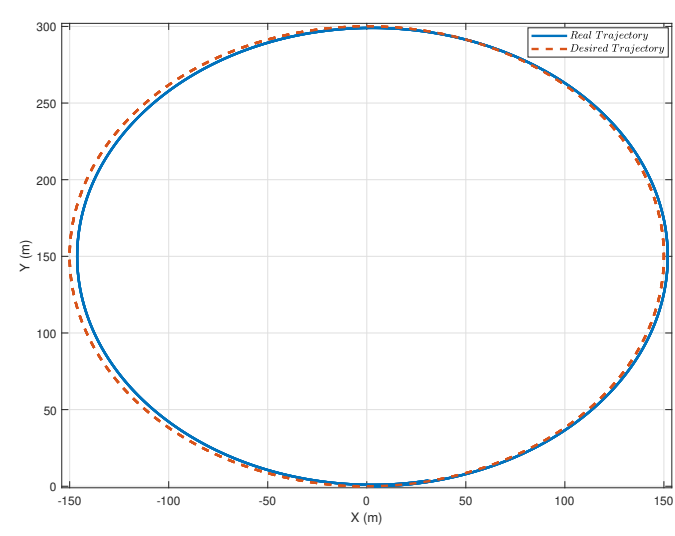

Fig. 15. Circular Path Following.
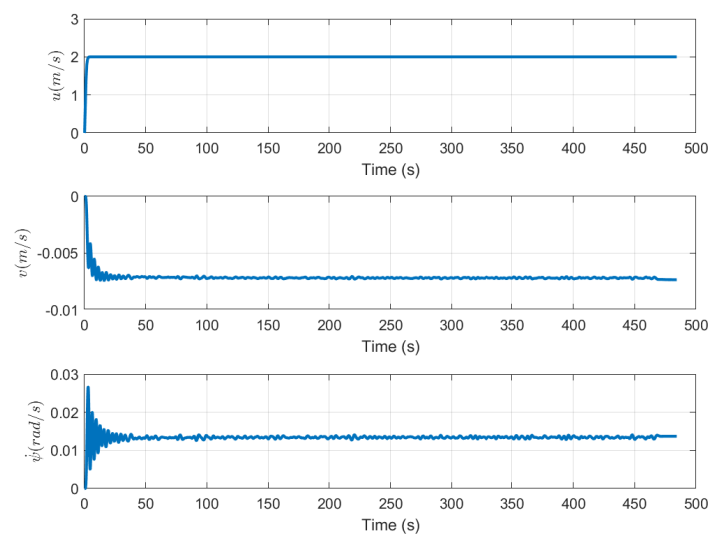

Fig. 16. Nonlinear Model Responses $u, v$ and $\dot{\psi}$ during circular path following.

the effort control level between its physical limitations. These initial results validate the proposed guidance and control schema to be implemented in the EDSON-J USV, which has the main mission to carry instrumentation and measure periodically water quality of the Peruvian sea.

\section{CONCLUSion}

A robust guidance control is presented for the EDSON$\mathrm{J}$, an unmanned surface vehicle under development at the

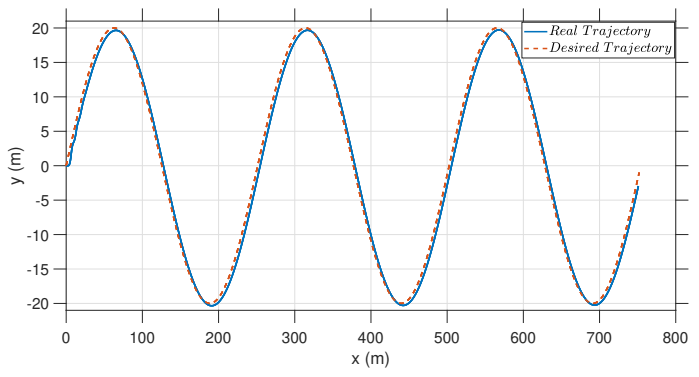

Fig. 17. Zigzag Path Following. 

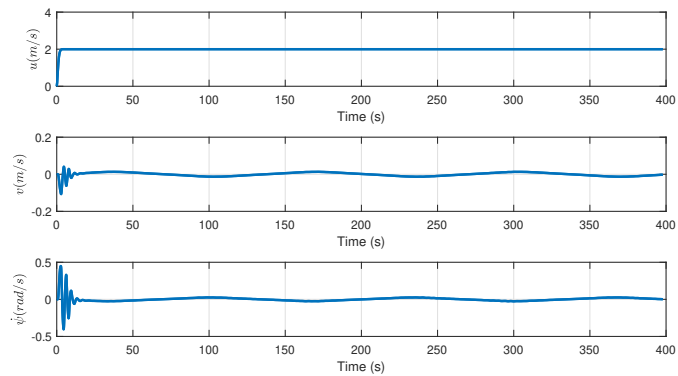

Fig. 18. Nonlinear Model Responses $u, v$ and $\dot{\psi}$ during Zigzag Path Following.

Universidad Nacional de San Agustín de Arequipa (Peru). The approaches are a Mamdani type Fuzzy logic guidance and an H-infinity mixed sensitivity robust control. To increase performance of the path following, the fuzzy logic approach received the steering command using the Lookahead-based algorithm. A suboptimal gamma of 1.0067 guaranteed control robustness in performance and in stability, and the line of sight ratio of $5 \mathrm{~m}$ guaranteed good guidance maneuvers considering a constant cruise speed of $2 \mathrm{~m} / \mathrm{s}$. This original guidance and control schema, validated numerically here, will serve to confront with experimental tests. Further works are in progress sensing and estimating the environmental disturbances in order to keep similar good performance at sea.

\section{ACKNOWLEDGMENT}

The authors thank the Universidad Nacional de San Agustin de Arequipa for the financial support given to this research, since 2019 and under grant number IBAIB-08-2018UNSA.

\section{REFERENCES}

[1] W. Elliott, R. Gonzales, N. Blas, A. Ramírez, C. Maldonado, M. Flores, and M. Jacinto, "Seguimiento de las pesquerías y calidad ambiental 2001-2005. imarpe huacho," Inf Inst Mar Perú, vol. 39, no. 1-2, 2012.

[2] J. Alves, P. Oliveira, R. Oliveira, A. Pascoal, M. Rufino, L. Sebastiao, and C. Silvestre, "Vehicle and mission control of the delfim autonomous surface craft," in 2006 14th Mediterranean Conference on Control and Automation. IEEE, 2006, pp. 1-6.

[3] S. Wirtensohn, J. Reuter, M. Blaich, M. Schuster, and O. Hamburger, "Modelling and identification of a twin hull-based autonomous surface craft," in 2013 18th International Conference on Methods \& Models in Automation \& Robotics (MMAR). IEEE, 2013, pp. 121-126.

[4] Y. Peng, Y. Yang, J. Cui, X. Li, H. Pu, J. Gu, S. Xie, and J. Luo, "Development of the usv 'jinghai-i' and sea trials in the southern yellow sea," Ocean engineering, vol. 131, pp. 186-196, 2017.
[5] C. Zhou, S. Gu, Y. Wen, Z. Du, C. Xiao, L. Huang, and $M$. Zhu, "The review unmanned surface vehicle path planning: Based on multi-modality constraint," Ocean Engineering, vol. 200, p. 107043, 2020. [Online]. Available: http://www.sciencedirect.com/science/article/pii/S0029801820301177

[6] J. C. Cutipa-Luque, D. C. Donha, J. L. D. Dantas, L. M. [de Oliveira], and E. A. [de Barros], "Robust control of an underactuated auv," IFAC Proceedings Volumes, vol. 45, no. 27, pp. 138 - 143, 2012, 9th IFAC Conference on Manoeuvring and Control of Marine Craft. [Online]. Available: http://www.sciencedirect.com/science/article/pii/S1474667016312186

[7] L. Qiao, S. Ruan, G. Zhang, and W. Zhang, "Robust h2 optimal depth control of an autonomous underwater vehicle with output disturbances and time delay," Ocean Engineering, vol. 165, pp. 399-409, 2018.

[8] C. Z. Ferreira, R. Cardoso, M. E. M. Meza, and J. P. J. Ávila, "Controlling tracking trajectory of a robotic vehicle for inspection of underwater structures," Ocean Engineering, vol. 149, pp. $373-382,2018$. [Online]. Available: http://www.sciencedirect.com/science/article/pii/S0029801817307540

[9] H. Zheng, J. Wu, W. Wu, and Y. Zhang, "Robust dynamic positioning of autonomous surface vessels with tube-based model predictive control," Ocean Engineering, vol. 199, p. 106820, 2020. [Online]. Available: http://www.sciencedirect.com/science/article/pii/S0029801819309187

[10] M. Breivik and T. I. Fossen, "Guidance laws for autonomous underwater vehicles," Underwater vehicles, vol. 4, pp. 51-76, 2009.

[11] J. C. C. Luque and D. C. Donha, "Auv robust guidance control," IFAC Proceedings Volumes, vol. 41, no. 1, pp. 85-90, 2008.

[12] D. Pearson, E. An, M. Dhanak, K. von Ellenrieder, and P. Beaujean, High-level fuzzy logic guidance system for an unmanned surface vehicle (USV) tasked to perform autonomous launch and recovery (ALR) of an autonomous underwater vehicle (AUV). IEEE, 2014.

[13] L. Wan, Y. Su, H. Zhang, B. Shi, and M. S. AbouOmar, "An improved integral light-of-sight guidance law for path following of unmanned surface vehicles," Ocean Engineering, vol. 205, p. 107302, 2020. [Online]. Available: http://www.sciencedirect.com/science/article/pii/S0029801820303437

[14] T. I. Fossen and A. M. Lekkas, "Direct and indirect adaptive integral line-of-sight path-following controllers for marine craft exposed to ocean currents," International Journal of Adaptive Control and Signal Processing, vol. 31, no. 4, pp. 445-463, 2017. [Online]. Available: https://onlinelibrary.wiley.com/doi/abs/10.1002/acs.2550

[15] W. Caharija, E. I. Grøtli, and K. Y. Pettersen, "Semiglobal exponential stability of a counter-current and co-current guidance scheme," IFAC-PapersOnLine, vol. 51, no. 29, pp. 274 - 280, 2018, 11th IFAC Conference on Control Applications in Marine Systems, Robotics, and Vehicles CAMS 2018. [Online]. Available: http://www.sciencedirect.com/science/article/pii/S2405896318322043

[16] B. Qiu, G. Wang, and Y. Fan, "Predictor los-based trajectory linearization control for path following of underactuated unmanned surface vehicle with input saturation," Ocean Engineering, vol. 214, p. 107874, 2020. [Online]. Available: http://www.sciencedirect.com/science/article/pii/S0029801820308416

[17] T. I. Fossen, Handbook of marine craft hydrodynamics and motion control. John Wiley \& Sons, 2011.

[18] J. C. Cutipa Luque, "Identificação e controle de um veículo submersível autônomo sub-atuado." Ph.D. dissertation, Universidade de São Paulo, 2012.

[19] S. Skogestad and I. Postlethwaite, Multivariable Feedback Control: Analysis and Design. John Whiley \& Sons, 2005. 